\title{
Feminilização de Larvas de Tilápia do Nilo (Oreochromis niloticus L.) a Partir de Banhos de Imersão com Valerato-de-Estradiol
}

\author{
Robie Allan Bombardelli ${ }^{1}$, Carmino Hayashi
}

\begin{abstract}
RESUMO - Determinou-se o período ontogênico de maior sensibilidade das larvas de tilápias do Nilo (Oreochromis niloticus) aos tratamentos de feminilização, por banhos de imersão de 36 horas, em solução contendo 2,0 mg de valerato-de-estradiol (VE). $\mathrm{L}^{-1}$. O experimento foi realizado em duas fases - a primeira com tratamentos hormonais e a segunda na fase de alevinagem. Foram utilizadas 1.200 larvas, provenientes de um mesmo lote de reprodutores, distribuídas em 24 recipientes plásticos de volume útil de 0,5 L, em um delineamento experimental inteiramente casualizado, composto por seis tratamentos e quatro repetições. Considerou-se a unidade experimental um recipiente plástico contendo $0,5 \mathrm{~L}$ de solução hormonal e 50 larvas. Os tratamentos constituíram-se no banho de imersão das larvas em diferentes fases ontogênicas, correspondentes a 175,2 UTAs (dias-grau) ou 6,5 DPE (dias após a eclosão); 217,2 UTAs ou 8 DPE; 273,2 UTAs ou 10 DPE; 329,0 UTAs ou 12 DPE; 383,9 UTAs ou 14 DPE; e 438,1 UTAs ou 16 DPE. Após os tratamentos, as larvas foram cultivadas até atingirem comprimento-padrão de $25,0 \mathrm{~mm}$, para posterior avaliação dos resultados. Os resultados de feminilização foram melhores para larvas mais jovens (6,5 DPE ou 175,2 UTAs), produzindo 39,20\% de fêmeas, o que demonstra relação linear negativa entre o período ontogênico e a taxa de feminilização. Os índices de comprimento e peso final médio, fator de condição e sobrevivência não foram afetados pelos tratamentos. O período ontogênico mais adequado para a reversão sexual com VE correspondeu àquele em que as larvas apresentaram 175,2 UTAs ou 6,5 DPE, o que produziu 39,20\% de fêmeas.
\end{abstract}

Palavras-chave: banho de imersão, estrógenos, ontogênese, Oreochromis niloticus, reversão sexual, tilápia

\section{Feminization of Larvae of Nile Tilapia (Oreochromis niloticus L.) by Immersion Baths with Estradiol Valerate}

\begin{abstract}
An experiment was carried out to in two steps (the first was hormonal treatments and the other the larvae grow up phase) to determine the best ontogenic periods for larvae of the Nile tilapia (Oreochromis niloticus) regarding the feminization hormonal treatments by immersion baths during 36 hours with a $2.0 \mathrm{mg}$ solution of estradiol valerate $17(\mathrm{VE}) . \mathrm{L}^{-1}$. One thousand and two hundred larvae of Nile tilapia, from the same reproduction bunch were used. Larvae were allotted to $240.5 \mathrm{~L}$ plastic containers in a completely randomized experimental design with six treatments and four replicates. An experimental unit consisted of a $0.5 \mathrm{~L}$ plastic container with hormonal solution and 50 larvae. Treatments comprised immersion of larvae at different ontogenic phases, or rather, 175.2 UTA (days - degrees) or 6.5 DAE (days after eclosion); 217.2 UTA or 8 DAE; 273.2. UTA or 10 DAE; 329.0 UTA or 12 DAE; 383.9 UTA or 14 DAE; and 438.1 UTA or 16 DAE. After treatments, the larvae were cultivated until they reached a standard length of $25 \mathrm{~mm}$ and thus their results could be estimated. Feminization results were best for younger larvae, with 6.5 DAE or 175.2 UTA, with $39.20 \%$ females. Results also suggested a negative linear relationship between the ontogenic period and feminization rates. There was no difference in treatments regarding indexes of average final length and weight, final condition factor and mortality. It was concluded that 175.2 UTA or 6.5 DAE is the best period for sexual reversion with $\mathrm{EV}$, with $39.20 \%$ females.
\end{abstract}

Key Words: immersion bath, estrogens, ontogenesis, Oreochromis niloticus, sexual reversion, tilapia

\section{Introdução}

O controle da diferenciação sexual, utilizando esteróides, tem sido realizado em diversas espécies de peixes teleósteos, em que os andrógenos levam à masculinização e estrógenos à feminilização (Yamamoto, 1969).
Apesar de a utilização dos estrógenos não ser amplamente discutida, estes hormônios têm sido utilizados em pelo menos 56 diferentes espécies de peixes, pertencentes a 24 famílias, das quais a maioria são gonocorísticas (Piferrer, 2001). Na aqüicultura, os principais interesses pela produção de fêmeas variam de acordo com a espécie cultivada, como as

\footnotetext{
1 Professor Assistente do Curso de Engenharia de Pesca da Universidade Estadual do Oeste do Paraná - UNIOESTE - Campus de Toledo. Rua da Faculdade, no 645 - Jardim La Salle - CEP: 85903-000, Toledo/PR. E.mail: rabombardelli@unioeste.br; rabombardelli@bol.com.br.

${ }^{2}$ Pesquisador do Centro Avançado de Pesquisa Tecnológica da Agronegócio de Pescado Continental, Instituto de Pesca, APTA;SAA São José do Rio Preto/SP. E.mail: hayashi@pesca.sp.gov.br.
} 
características ornamentais (George \& Pandian, 1995), maiores taxas de crescimento que os machos e controle da maturidade sexual (Goetz et al., 1979; Strussmann et al., 1996; Hendry et al., 2003; Specker $\&$ Chandlee, 2003) e até mesmo como modelos de estudo dos mecanismos endócrinos da diferenciação sexual, em espécies hermafroditas (Lee et al., 2001).

Atualmente, as tilápias é um dos grupos de peixes de maior potencial para a piscicultura (Stickney, 2000) em países de clima tropical e subtropical (Campos-Ramos et al., 2003; Desprez et al., 2003).

Em virtude da rápida taxa de crescimento, da adaptabilidade às diversas condições de cultivo (Hayashi, 1995; Boscolo et al., 2001) e da grande aceitação pelo mercado consumidor, as espécies do gênero Oreochromis, em especial Oreochromis niloticus (Lovshin, 1998), são consideradas uma das mais importantes para as condições de cultivo (Meurer et al., 2000). Isso justifica a importância desta espécie, que é a segunda mais cultivada no mundo (Lovshin, 1997; Alceste \& Jorry, 1998) e a primeira no Brasil (Lovshin \& Cyrino, 1998).

Na tilapicultura, utilizam-se comumente populações monosexuais masculinas (Phelps \& Popma, 2000; Beardmore et al., 2001), produzidas com o uso de andrógenos (Penman \& McAndrew, 2000), incorporados na ração (MacIntosh \& Little, 1995; Leonhardt, 1997), visando minimizar os problemas relacionados à reprodução e às taxas de crescimento.

Apesar de o uso de andrógenos para a masculinização ser uma técnica difundida e de os hormônios esteróides (estrógenos e andrógenos) pesquisados serem rapidamente metabolizados e excretados (Johnstone et al., 1983; Pillay, 1990; Hendry et al., 2003; Specker \& Chandlee, 2003), constantes questionamentos são feitos quanto a seus possíveis efeitos na saúde humana (Piferrer, 2001).

A produção de fêmeas na tilapicultura pode ser realizada com o objetivo de feminilização de machos genéticos (XY) para a posterior produção de machos homogaméticos (YY) (Baldisserotto, 2002), conhecidos comumente como "super-machos", em programas de cruzamento (Pandian \& Sheela, 1995; Donaldson, 1996; Phelps \& Popma, 2000). "Super-machos", quando cruzados com fêmeas normais, produzem, teoricamente, proles $100 \%$ masculinas (Penman \& McAndrew, 2000), sem o uso de hormônios.

A feminilização, além de ser um procedimento técnico, pode ser uma estratégia de marketing (Popma \& Phelps, 1998) e/ou alternativa às proibições pelas legislações de determinados países (Desprez et al., 1995), para a produção de proles monossexuais masculina com o uso de hormônios esteróides (Scott et al., 1989; Mélard, 1995).

A incorporação de estrógenos na ração é uma técnica eficiente na feminilização de Oreochromis aureus (Mélard, 1995) e Odontesthes bonariensis (Strussmann et al., 1996). Contudo, existe tendência de redução donível de intervenção dos tratamentos hormonais no controle da diferenciação sexual (Piferrer, 2001).

Atualmente, a técnica de banhos de imersão não é comercialmente empregada, em decorrência da falta de protocolo específico. Assim, desde que seja previamente conhecido o período de maior sensibilidade aos tratamentos com hormônios exógenos (Piferrer, 2001), esta técnica pode ser uma alternativa vantajosa, visto que, além do menor nível de intervenção, pode apresentar menor custo (Pandian \& Sheela, 1995) e maior controle sobre variáveis como a taxa de obtenção de alimento e as hierarquias sociais (Gale et al., 1999; Beardmore et al., 2001). Além disso, a imersão poderá reduzir a exposição das pessoas que manipulam o hormônio e ser ambientalmente mais segura, por possibilitar o armazenamento do resíduo para posterior degradação (Gale et al., 1999) ou filtragem em carbono ativado (Specker \& Chandlee, 2003).

Objetivou-se, com o presente trabalho, determinar a fase ontogênica de maior sensibilidade ao tratamento para feminilização com o estrógeno valerato-de-estradiol, a partir de banhos de imersão, e avaliar seus efeitos sobre o peso, comprimento, fator de condição e sobrevivência em larvas de tilápia do Nilo (Oreochromis niloticus).

\section{Material e Métodos}

O presente experimento foi realizado no Laboratório de Aqüicultura do Departamento de Biologia da Universidade Estadual de Maringá, com início em 22/01/2003 e término em 03/03/2003, totalizando 40 dias. O trabalho foi realizado em duas fases - a primeira foi constituída pelos tratamentos de imersão, em solução hormonal, das larvas em diferentes fases de desenvolvimento ontogênico, e a segunda, pela fase de crescimento ou alevinagem dos animais até atingirem o comprimento-padrão mínimo para avaliação dos índices de desempenho e sobrevivência.

Foram utilizadas 1.200 larvas de tilápia do Nilo (Oreochromis niloticus), da linhagem tailandesa, 
provenientes de diversas proles de um mesmo lote de reprodutores. Inicialmente, foi retirada uma amostra de 100 larvas para a determinação das médias do peso e comprimento inicial. Em seguida, as larvas foram todas alojadas em 24 gaiolas $(1,5 \mathrm{~L})$, a uma densidade de 50 larvas por gaiola. Cada gaiola foi instalada dentro de um tanque-rede experimental de malha $1,0 \mathrm{~mm}$ e dimensões de $0,4 \mathrm{~m}$ x 0,5 m x 0,6 m, que estavam distribuídos dentro de quatro caixas de fibro-cimento de volume de $1.000 \mathrm{~L}$ (Figura 1). As gaiolas receberam aeração por pedra micro porosa e as caixas de $1.000 \mathrm{~L}$ receberam circulação constante de água, proporcionando uma taxa de renovação de aproximadamente $20 \%$ do volume total ao dia. Este procedimento teve duração até a realização do último tratamento hormonal e foi realizado para manter a homogeneidade entre as densidades de estocagem.

Na primeira fase, os animais foram transferidos das gaiolas para recipientes de plásticos, de volume útil de $0,5 \mathrm{~L}$, sendo distribuídos em um delineamento experimental inteiramente casualizado, composto por seis tratamentos hormonais e quatro repetições, totalizando 24 unidades experimentais. Foi considerada como uma unidade experimental, um recipiente plástico contendo um volume útil de solução de $0,5 \mathrm{~L}$ e 50 larvas em cada. Os seis tratamentos foram constituídos pela imersão das larvas em diferentes idades, correspondentes a 175,2 UTAs (dias - grau) ou 6,5 DPE (dias após a eclosão); 217,2 UTAs ou 8 DPE; 273,2 UTAs ou 10 DPE; 329,0 UTAs ou 12 DPE; 383,9 UTAs ou 14 DPE; e 438,1 UTAs ou 16

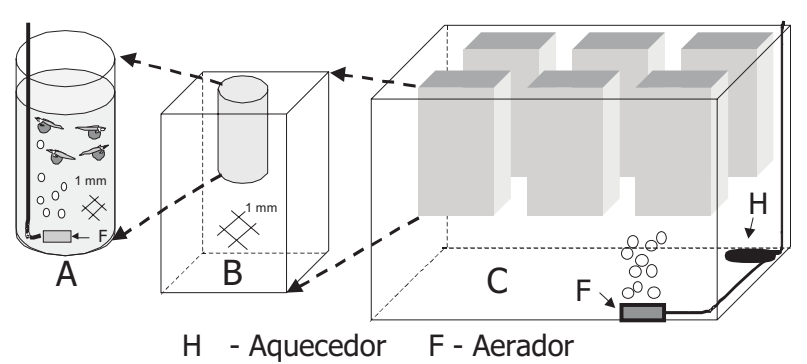

Figura 1 - Desenho esquemático das instalações utilizadas para alojar os animais durante 0 período experimental. A - Gaiola de 1,5 L de $1000 \mathrm{~L}, \mathrm{~B}$ - Tanque-rede experimental $(0,4 \mathrm{~m}$ X 0,5 m X 0,6 m) e C - Caixa de fibro - cimento de $1.000 \mathrm{~L}$.

Figure 1 - Installations schematical drawing used to lodge the animals during the experimental period. $A-1.5 \mathrm{~L}$ cage; $B$ - Experimental net pond $(0.4 \mathrm{~m} \times 0.5 \mathrm{~m} \times$ $0.6 \mathrm{~m})$, and $C-1,000 \mathrm{~L}$ water box.
DPE. O estrógeno utilizado foi o valerato-de-estradiol e o veículo de diluição o álcool etílico $\left(95^{\circ} \mathrm{GL}\right)$. Baseado em ensaios preliminares, em todos os tratamentos os banhos tiveram a duração de 36 horas e concentração hormonal de $2,0 \mathrm{mg}$ de valerato-deestradiol.L $\mathrm{L}^{-1}$ de solução. Procurou-se manter uma concentração alcoólica final da solução de 0,04\% (Goetz, et al., 1979), não prejudicial aos peixes.

Durante os tratamentos hormonais, as unidades experimentais receberam aeração por pedra micro porosa, para possibilitar a constante homogeneização da solução (Gale et al., 1999), e evitar a hipoxia. Estas unidades foram mantidas em um tanque de concreto com aproximadamente $400 \mathrm{~L}$, em forma de banho-maria, a fim de permitir a homogeneização da temperatura entre as unidades experimentais (Figura 2).

O tanque de concreto utilizado na fase I foi dotado de sistema de aquecimento por resistência elétrica de $2.000 \mathrm{~W}$, ligada a um termostato, mantendo-se a temperatura mínima e máxima entre 27,0 e $28,5^{\circ} \mathrm{C}$, respectivamente. Durante os tratamentos, a temperatura da água foi aferida a partir de um termômetro de máxima e mínima, com escala de precisão de $1^{\circ} \mathrm{C}$.

Após os banhos de imersão, durante a alevinagem (fase II), as larvas permaneceram dentro das gaiolas, sendo liberadas nos respectivos tanques-rede de cada tratamento, somente após o último tratamento hormonal (16 DPE). Nesta fase, os animais foram distribuídos novamente em delineamento experimental em blocos inteiramente casualizado, composto por

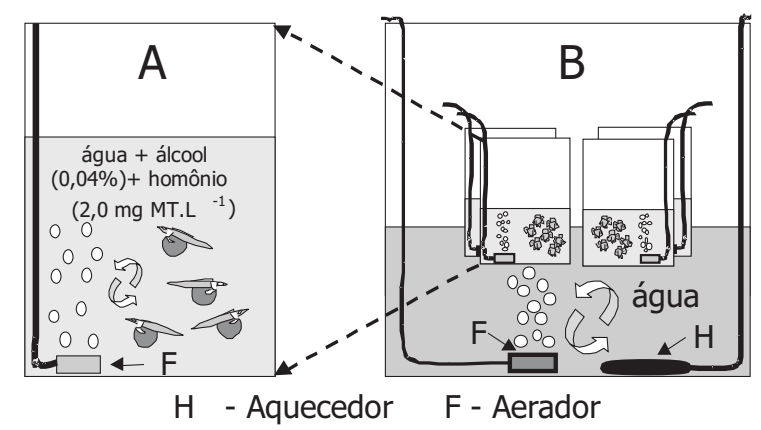

Figura 2 - Desenho esquemático das estruturas utilizadas para a realização dos tratamentos hormonais. A - Recipiente plástico de $0,5 \mathrm{~L}$ e B - Tanque de concreto de $400 \mathrm{~L}$.

Figure 2 - Structrure schematical drawing used to accomplish hormonal treatments. $A-0.5 L$ plastic container and $B$ - $400 L$ water box.

R. Bras. Zootec., v.34, n.2, p.357-364, 2005 
seis tratamentos e quatro repetições. Foi considerada como uma unidade experimental, um tanque-rede, contendo 50 larvas e os blocos constituídos por uma caixa de fibro-cimento de volume útil de $1.000 \mathrm{~L}$, contendo seis unidades experimentais, referentes a cada tratamento.

Os tanques-rede utilizados na fase II receberam aeração por pedra micro porosa, e a temperatura da água das caixas foi controlada com a utilização de resistências elétricas, de modo a manter este parâmetro por volta de 26,0 a $28,0^{\circ} \mathrm{C}$, evitando assim a masculinização devido à exposição as altas temperaturas (Baroiller \& D’Cotta, 2001).

Os animais foram cultivados até atingirem o comprimento padrão mínimo de $25,0 \mathrm{~mm}$. As larvas foram alimentadas, diariamente, à vontade, com uma freqüência de arraçoamento de 5 vezes ao dia (Sanches \& Hayashi, 2001), com uma ração-base, farelada (Meurer et al., 2003) e sem hormônio (Tabela 1), atendendo às exigências nutricionais da espécie (Hayashi et al., 2002), com 38,5\% de proteína digestível e $3.800 \mathrm{kcal}$ de energia digestível. $\mathrm{kg}^{-1}$ de ração. Os alimentos utilizados na formulação da ração foram processados de acordo com Hayashi et al. (1999).

Diariamente, pela manhã e pela tarde, foi mensurada a temperatura da água $\left({ }^{\circ} \mathrm{C}\right)$, por intermédio de um termômetro de mercúrio, escala de precisão de $1^{\circ} \mathrm{C}$, e realizada a sifonagem da água das caixas e limpeza das telas dos "hapas", para retirada das excretas e restos de alimento. Semanalmente, foram mensurados o oxigênio dissolvido $\left(\mathrm{mg} . \mathrm{L}^{-1}\right)$, a condutividade elétrica $\left(\mu \mathrm{S} . \mathrm{cm}^{-1}\right)$ e o $\mathrm{pH}$ da água.

Ao término do experimento, os alevinos foram dessensibilizados por choque térmico, a aproximadamente $2^{\circ} \mathrm{C}$ e, em seguida, sacrificados para a mensuração do comprimento $(\mathrm{mm})$ e peso (mg) médios final. O fator de condição e a taxa de sobrevivência (\%) também foram calculados.

Em seguida, os animais foram fixados inteiros em solução de formalina a $10 \%$, para análise sob microscopia óptica, das gônadas coradas, segundo Popma \& Green (1990), para a determinação da proporção entre os sexos.

O modelo estatístico utilizado para as análises das variáveis estudadas foi:

$$
Y_{i j}=\beta_{o}+\beta_{1} x_{i}+\beta_{2} x_{2}+e_{i j}
$$

em que $Y_{i j}=$ observação referente ao recipiente j em que se utilizou o período ontogênico i; $\beta_{o}=$ constante
Tabela 1 - Composição percentual da ração-base utilizada durante o período experimental

Table 1 - Percentage composition of the ration used during the experimental period

\begin{tabular}{|c|c|}
\hline $\begin{array}{l}\text { Alimento } \\
\text { Food }\end{array}$ & $\begin{array}{r}\text { Quantidade (\%) } \\
\text { Amount (\%) }\end{array}$ \\
\hline Farinha de vísceras de frango & 54,63 \\
\hline Poultry viscera meal ${ }^{1}$ & \\
\hline $\begin{array}{l}\text { Farelo de soja } \\
\text { Soybean meal }\end{array}$ & 19,15 \\
\hline $\begin{array}{l}\text { Levedura } \\
\text { spray dried spray dries yeast } 1\end{array}$ & 6,00 \\
\hline $\begin{array}{l}\text { Milho } \\
\text { Corn }^{2}\end{array}$ & 7,68 \\
\hline $\begin{array}{l}\text { Óleo de soja } \\
\text { Soybean oil }\end{array}$ & 10,02 \\
\hline $\begin{array}{l}\text { Suplemento }(\min +v i t) \\
\text { Supplement }(\min +v i t)\end{array}$ & 2,00 \\
\hline $\begin{array}{l}\text { Sal } \\
\text { Salt }\end{array}$ & 0,50 \\
\hline $\begin{array}{l}\text { Antioxidante BHT } \\
\text { Antioxidant BHT }\end{array}$ & 0,02 \\
\hline
\end{tabular}

${ }^{1}$ De acordo com os valores de digestibilidade de Meurer (2002).

2 De acordo com os valores de digestibilidade de Boscolo et al. (2002).

${ }_{1}$ According to the digestibility values from Meurer (2002).

${ }^{2}$ According to the digestibility values from Boscolo et al. (2002).

geral; $\beta_{1}=$ coeficiente linear de regressão da variável Y em função do período ontogênico i; $\beta_{2}=$ coeficiente quadrático de regressão da variável $\mathrm{Y}$ em função do período ontogênico i; $x_{i}=$ período ontogênico $\mathrm{i} ; e_{i j}=$ erro aleatório associado a cada observação $Y_{i j}$.

Os dados de proporção entre os sexos, de peso, comprimento, fator de condição e sobrevivência, bem como dos parâmetros físico-químicos da água, foram submetidos à análise de variância ao nível de 5\% de probabilidade e, nos casos da evidência de diferença significativa, aplicou-se a análise de regressão. O software utilizado para realização das análises estatísticas foi o Sistema de Análise Estatística e Genética - SAEG (UFV, 1997).

\section{Resultados e Discussão}

Nos diferentes tratamentos, os valores médios de temperatura, $\mathrm{pH}$, oxigênio dissolvido e condutividade elétrica da água, não apresentaram diferença $(\mathrm{P}>0,05)$, sendo de 27,39 $\pm 1,18^{\circ} \mathrm{C} ; 7,89 \pm 0,43 ; 7,39 \pm 0,31 \mathrm{mg} . \mathrm{L}^{-1}$; $0,18 \pm 0,01 \mu \mathrm{S} . \mathrm{cm}^{-1}$, respectivamente, mantendo-se dentro dos limites considerados recomendáveis para o cultivo de peixes (Boyd, 1990; Sipaúba-Tavares, 
1995) e para o bom desempenho da espécie (Popma \& Phelps, 1998).

O percentual de fêmeas variou entre 24,05 e 39,20 (Tabela 2), sugerindo a existência de um período de maior sensibilidade das larvas aos tratamentos por imersão, com estrógeno valerato-de-estradiol, aos 175,2 UTAs ou 6,5 DPE. Os resultados demonstram uma relação linear negativa $(\mathrm{P}<0,05)$, com relação ao período ontogênico e o grau de feminilização (Figura 3). $O$ percentual de indivíduos interssexuais não foi afetado pelos tratamentos $(\mathrm{P}>0,05)$ (Tabela 2). Neste caso, foi constatada relação linear positiva $(\mathrm{P}<0,05)$ entre a idade e o percentual de machos.

As evidências da existência de um período de maior sensibilidade aos tratamentos hormonais com estrógenos e andrógenos, têm sido constatadas em diferentes espécies como Oncorhynchus kisutch (Piferrer \& Donaldson, 1989), Oncorhynchus mykiss (Krisfalusi \& Nagler, 2000), Oryzias latipes (Koger et al., 2000) e Oreochromis niloticus (Apple \& Leboute, 1995).

Os resultados de feminilização, ao contrário dos tratamentos com andrógenos, mostram uma melhor resposta nas larvas mais jovens. Em Oncorhynchus kisutch, a sensibilidade ao $17 \mathrm{~b}$-estradiol ocorre alguns dias antes (aproximadamente 12 dias) que para a $17 \alpha$-metiltestosterona (Piferrer \& Donaldson, 1989).

Os resultados neste experimento, sugerem o mesmo comportamento para a tilápia do Nilo, tendo havido melhor efetividade aos tratamentos com estrógeno para larvas mais jovens (Tabela 2). Por outro lado, larvas de maior idade, da mesma espécie,

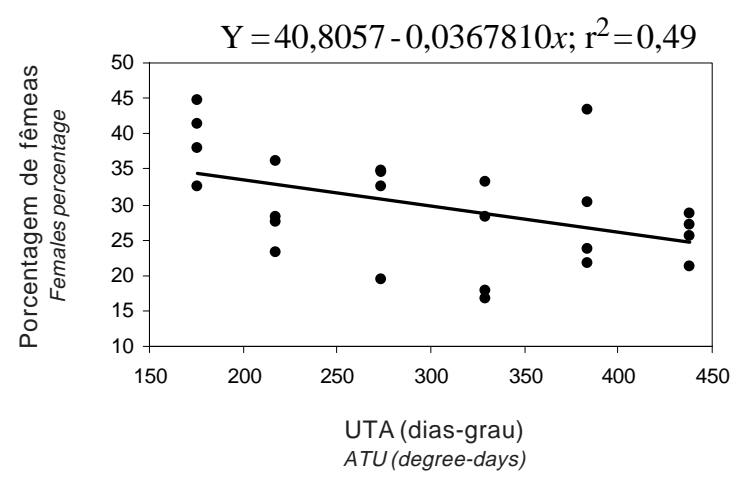

Figura 3 - Porcentagem de feminilização de larvas de tilápias do Nilo, submetidas a banhos de imersão com valerato de estradiol, em diferentes períodos ontogênicos.

Figure 3 - Percentage of feminilization of larvae of Nile tilapia, submitted to bath imersion with estradiol valerate, in different ontogenic stage. submetidas a $17 \alpha$-metiltestosterona e androstenediona, no 70 e 14음 DPE, resultaram na melhor resposta (Apple \& Leboute, 1995).

Estes resultados podem ser comprovados, pelas evidências histológicas da completa diferenciação sexual nas fêmeas ocorrer antes que nos machos (Strussmann et al., 1996; Piferrer, 2001).

Ao contrário dos andrógenos, a obtenção do sucesso dos tratamentos com estrógenos parece ser mais difícil de ser alcançado (McAndrew, 1993), porque os andrógenos são agentes alteradores de sexo mais potentes que os estrógenos, fato que leva à necessidade da utilização de maiores dosagens hormonais para feminilizar efetivamente os peixes (Hendry et al., 2003). Contudo, mesmo utilizando altas dosagens, em algumas espécies como as carpas, o emprego de $17 \beta$-estradiol foi completamente ineficiente (Komen \& Richter, 1993). Esta dificuldade em obter a feminilização parece se agravar em procedimentos que utilizem banhos de imersão, chegando a serem não efetivos (Phelps \& Popma, 2000).

Os resultados de $24,05 \%$ a $39,20 \%$ de feminilização, obtidas neste experimento (Tabela 2), confirmaram as afirmações referentes à ineficiência dos tratamentos, principalmente quando são comparados com resultados de outros autores. Mélard (1995), obteve $100 \%$ de feminilização utilizando suplementação dietética de $200 \mathrm{mg}$ de 17 aetinilestradiol. $\mathrm{kg}^{-1}$ na dieta, em $O$. aureus. Scott et al. (1989) obtiveram bons resultados de feminilização em proles de $O$. niloticus, geneticamente monossexuais masculinas. Estes autores alcançaram resultados de 51,6 e $83,9 \%$ em fêmeas, em dois ensaios, respectivamente, utilizando $100 \mathrm{mg}$ de dietilstilbestrol. $\mathrm{kg}^{-1}$ de dieta.

Neste experimento, as reduzidas taxas de feminilização obtidas (Tabela 2) não podem ser atribuídas ao "efeito paradoxal de masculinização", contrariando o "efeito paradoxal de feminilização", que é evidenciado pela aplicação de altas dosagens de andrógenos (Piferrer \& Donaldson, 1991), uma vez que estes não têm sido constatados em peixes (Piferrer, 2001). Assim, estes resultados podem ser explicados pelo sistema de determinação sexual característico da tilápia do Nilo (Oreochromis niloticus), proveniente da linhagem tailandesa, onde a proporção entre os sexos pode ser desviada de 1:1, favorecendo a produção natural de maior percentual de machos. Tuan et al. (1999) verificaram que, apesar de a proporção geral entre os sexos das progênies da

R. Bras. Zootec., v.34, n.2, p.357-364, 2005 
Tabela 2 - Valores médios de desempenho zootécnico e proporção entre os sexos de larvas de tilápia do Nilo (Oreochromis niloticus), submetidas a banhos de imersão com valerato-de-estradiol, em diferentes períodos ontogênicos

Table 2 - Values of performance and sexual ratio of larvae of Nile tilapia (Oreochromis niloticus), submitted to bath immersion with estradiol valerate, in different ontogenic period

\begin{tabular}{|c|c|c|c|c|c|c|c|}
\hline \multirow[b]{2}{*}{$\begin{array}{l}\text { Resultado } \\
\text { Result }\end{array}$} & \multicolumn{6}{|c|}{$\begin{array}{l}\text { Unidade térmica acumulada / UTA (dias - grau) } \\
\text { Accumulated thermal unit / ATU (degree - days) }\end{array}$} & \multirow[b]{2}{*}{$\mathrm{CV}$} \\
\hline & 175,2 & 217,2 & 273,2 & 329,0 & 383,9 & 438,1 & \\
\hline $\begin{array}{l}\text { Peso inicial }(\mathrm{mg}) \\
\text { Initial weight }(\mathrm{mg})\end{array}$ & 9,32 & 12,22 & 15,82 & 20,91 & 25,91 & 32,52 & \\
\hline $\begin{array}{l}\text { Peso final }(\mathrm{mg}) \\
\text { Final weight }(\mathrm{mg})\end{array}$ & 900,00 & 910,00 & 850,00 & 910,00 & 900,00 & 800,00 & $9,71^{\mathrm{ns}}$ \\
\hline $\begin{array}{l}\text { Comprimento inicial }(\mathrm{mm}) \\
\text { Initial lenght }(\mathrm{mm})\end{array}$ & 0,81 & 0,91 & 1,08 & 1,17 & 1,18 & 1,32 & \\
\hline $\begin{array}{l}\text { Comprimento final }(\mathrm{mm}) \\
\text { Final lenght }(\mathrm{mm})\end{array}$ & 28,32 & 28,12 & 27,30 & 27,37 & 27,87 & 27,15 & $3,93^{\mathrm{ns}}$ \\
\hline $\begin{array}{l}\text { Fator de condição } \\
\text { Condictionfactor }\end{array}$ & 0,040 & 0,041 & 0,042 & 0,044 & 0,042 & 0,040 & $7,58^{\text {ns }}$ \\
\hline $\begin{array}{l}\text { Sobrevivência (\%) } \\
\text { Survival (\%) }\end{array}$ & 95,50 & 92,00 & 93,50 & 89,00 & 90,50 & 96,00 & $5,50^{\mathrm{ns}}$ \\
\hline $\begin{array}{l}\text { Machos }(\%)^{1} \\
\text { Male sex ratio (\%) }\end{array}$ & 49,32 & 60,58 & 62,17 & 66,48 & 66,69 & 65,48 & $11,09^{*}$ \\
\hline $\begin{array}{l}\text { Fêmeas }(\%)^{2} \\
\text { Female sex ratio }(\%)\end{array}$ & 39,20 & 28,84 & 30,45 & 24,05 & 29,81 & 25,68 & $23,09^{*}$ \\
\hline $\begin{array}{l}\text { Interssexuais }(\%) \\
\text { Intersex sex ratio (\%) }\end{array}$ & 11,48 & 10,59 & 7,39 & 9,47 & 3,49 & 8,83 & $45,54^{\mathrm{ns}}$ \\
\hline
\end{tabular}

* Dados com diferenças significativas a $5 \%$ de probabilidade.

* Data with significant difference at $5 \%$ of probability.

ns Dados sem diferenças significativas.

linhagem tailandesa não diferir de 1:1, existe grande variação desta taxa entre diferentes grupos de reprodutores. Esses autores observaram que, entre os grupos avaliados, $53,7 \%$ apresentaram proporção entre os sexos diferente de $1: 1$, sendo que destes $28,4 \%$ desviavam a proporção entre os sexos para machos e $25,3 \%$ para fêmeas. Considerando que Bombardelli (2002) (Comunicação pessoal) verificou em ensaios preliminares que as proporções sexuais encontradas para tilápias desta linhagem, cultivadas sob temperaturas de $25,0 \mathrm{e}$ $28,0^{\circ} \mathrm{C}$, foram de 74,2 e $73,7 \%$ de machos, respectivamente, pode-se inferir que os tratamentos de feminilização foram praticamente ineficientes. Outro fator determinante na ineficiência dos tratamentos de feminilização pode ter sido a falta de ajuste das dosagens hormonais, que foram reduzidas.

Em relação aos parâmetros das médias de peso final ( $\mathrm{mg}$ ), comprimento final $(\mathrm{cm})$, fator de condição e sobrevivência (\%), não foram observadas diferenças significativas $(\mathrm{P}>0,05)$ entre os tratamentos.
Koger et al. (2000), ao estudarem o estádio de maior sensibilidade em Oryzias latipes, à testosterona e $17 \beta$-estradiol, não verificaram diferenças significativas quanto à mortalidade e peso corporal. Mélard (1995) também verificou altas taxas de sobrevivência (97\%) de larvas de Oreochromis aureus, submetidas a tratamentos com estrógeno a partir de suplementação dietética. Apesar desses resultados e do presente experimento não indicarem a existência de toxidade dos tratamentos, evidenciada por Phelps \& Popma (2000), este efeito pode não ter sido verificado, em decorrência das reduzidas dosagens hormonais. A ausência de significância $(P>0,05)$ entre os tratamentos para peso final, comprimento final e fator de condição pode ser explicada pelo fato de as dosagens hormonais serem iguais para todas as idades, associadas à curta duração dos banhos.

A partir destes resultados, novos experimentos deverão ser realizados para ajustes de variáveis como dose, duração dos tratamentos e densidades, a fim de maximizar a eficiência dos tratamentos. 


\section{Conclusões}

A feminilização por banhos de imersão contendo valerato-de-estradiol sofreu decréscimo linear, em função do período ontogênico das larvas de tilápia da Nilo (Oreochromis niloticus), observando-se maior sensibilidade aos 175,2 UTAs ou 6,5 DPE, com $39,20 \%$ de fêmeas. Os índices zootécnicos de desempenho e sobrevivência não foram afetados pelos tratamentos.

\section{Literatura Citada}

ALCESTE, C.; JORRY, D.E. Análisis de las tendencias actuales en la comercialización de tilapia en los Estados Unidos de Norteamérica y la Unión Europea. In: AQUICULTURA BRASIL, 10., 1998, Recife. Anais... Recife: Associação Brasileira de Aquicultura, 1998. p.349-364.

APPLE, H.B.; LEBOUTE, E.M. Masculinização de pós-larvas de tilápia do Nilo (Oreochromis niloticus) utilizando andrógenos através de tratamentos de imersão. In: ENCONTRO SUL BRASILEIRO DE AQUICULTURA, 3., 1995, Ibirubá. Anais... Ibirubá: COTRIBÁ e Universidade Federal do Rio Grande do Sul, 1995. p.113-119.

BALDISSEROTTO, B. Fisiologia de peixes aplicada à piscicultura. Santa Maria: Universidade Federal de Santa Maria, 2002. 211p.

BAROILLER, J.F.; D'COTTA, H. Environment and Sex determination in farmed fish. Comparative Biochemistry and Physiology Part C, v.130, p.399-409, 2001.

BEARDMORE, J.A.; MAIR, G.C.; LEWIS, R.I. Monosex male production in finfish as exemplified by tilapia: applications, problems and prospects. Aquaculture, v.197, p.283-301, 2001.

BOSCOLO, W.R.; HAYASHI, C.; MEURER, F. Digestibilidade aparente da energia e nutrientes de alimentos convencionais e alternativos para o tilápia do Nilo (Oreochromis niloticus). Revista Brasileira de Zootecnia, v.31, n.2, p.539-545, 2002.

BOSCOLO, W.R.; HAYASHI, C.; SOARES, C.M. et al. Desempenho e características de carcaça de machos revertidos de Tilápias do Nilo (Oreochromis niloticus), linhagens tailandesa e comum, nas fases inicial e de crescimento. Revista Brasileira de Zootecnia, v.30, n.5, p.1391-1396, 2001.

BOYD, C.E. Water quality in ponds for aquaculture. Alabama: Birmingham Publishing Co, 1990. 482p.

CAMPOS-RAMOS, R.; HARVEY, S.C.; MCANDREW, B.J. et al. An invetigation of sex determination in the Mozambique tilapia, Oreochromis mossambicus, using synaptonemal complex analysis, FISH, sex reversal and gynogenesis. Aquaculture, v.221, p.125-140, 2003.

DESPREZ, D.; GÉRAZ, E.; HOAREAU, M.C. et al. Production of a high percentage of male offspring with a natural androgen, $11 \mathrm{~b}$ - hydroxyandrostenedione (11bOHA4), in Florida red tilapia. Aquaculture, v.216, p.55-65, 2003.

DESPREZ, D.; MÉLARD, C.; PHILIPPART, J. C. Production of a high percentage of male offspring with $17 \alpha$ - ethynylestradiol sex - reversed Oreochromis aureus. II. Comparative reproductive biology of females and F2 pseudofemales and large-scale production of male progeny. Aquaculture, v.130, p.35-41, 1995.

R. Bras. Zootec., v.34, n.2, p.357-364, 2005
DONALDSON, E.M. Manipulation of reproduction in farmed fish. Animal Reproduction Science, v.42, p.381-392, 1996.

GALE, W.L.; FITZPATRICK, M.S.; LUCERO, M. et al. Masculinization of Nile tilapia (Oreochromis niloticus) by immersion in androgens. Aquaculture, v.178, p.349-357, 1999.

GEORGE, T.; PANDIAN, T.J. Production of ZZ females in the female - heterogametic black molly, Poecilia sphenops, by endocrine sex reversal and progeny testing. Aquaculture, v.136, p.81-90, 1995.

GOETZ, F. W.; DONALDSON, E.M.; HUNTER, G.A. et al. Effects of estradiol $17 \beta$ and $17 \alpha$ methyltestosterone on gonadal differentiation in the coho salmon, Oncorhynchus kisutch. Aquaculture, v.17, p.267-278, 1979.

HAYASHI, C. Breves considerações sobre as tilápias In: RIBEIRO, R.P.; HAYASHI, C.; FURUYA, W.M. (Eds.) Curso de piscicultura: criação racional de tilápias. Maringá: EDUEM, 1995. p.4.

HAYASHI, C.; BOSCOLO, W.R.; SOARES, C.M. et al. Exigência de proteína digestível para larvas de tilápia do Nilo (Oreochromis niloticus) durante a reversão sexual. Revista Brasileira de Zootecnia, v.31, n.2, p.823-829, 2002.

HAYASHI, C.; BOSCOLO, W.R.; SOARES, C.M. et al. Uso de diferentes graus de moagem dos ingredientes em dietas para tilápia do Nilo (Oreochromis niloticus $L$.) na fase de crescimento. Acta Scientiarum, v.21, n.3, p.733-737, 1999.

HENDRY, C.I.; MARTIN-ROBICHAUD, D.J.; BENFEY, T.J. Hormonal sex reversal of Atlantic halibut (Hippoglossus hippoglossus L.). Aquaculture, v.219, p.769-781, 2003.

JOHNSTONE, R.; MACINTOSH D.J.; WRIGHT, R.S. Elimination of orally administered 17 methyl testosterone by Oreochromismossambicus (tilapia) and Salmo gaidneri (rainbow trout) juveniles. Aquaculture, v.35, p.249-257, 1983.

KOGER, C.S.; TEH, S.J.; HINTON, D.E. Determining the sensitive developmental stages of intersex induction in medaka (Oryzias latipes) exposed to 17 beta- estradiol or testosterone. Marine Environmental Research, v.50, n.1-5, p.201-206, 2000.

KOMEN, J.; RICHTER, C.J. Sex control in carp In: MUIR, J.F.; ROBERTS, R.J. (Eds.) Recent advances in aquaculture IV. London: Blackwell Scientific Plublications, 1993. p.78-86.

KRISFALUSI, M.; NAGLER, J.J. Induction of gonadal intersex in genotypic male rainbow trout (Oncorhynchus mykiss) embryos following immersion in estradiol-17beta. Molecular Reproduction and Development, v.56, n.4, p.495-501, 2000.

LEE, Y.H.; DU, J.L.; YUEH, W.S. et al. Sex change in protandrous black porgy, Acanthopagrus schlegeli: A review in gonadal development, estradiol, estrogen receptor, aromatase receptor, aromatase activity and gonadotropin. The Journal of Experimental Zoology, v.290, n.7, p.715-726, 2001.

LEONHARDT, J.H. Efeito da reversão sexual em tilápia do Nilo, Oreochromis niloticus (Linnaeus, 1757). Jaboticabal: Universidade Estadual Paulista, 1997. 128p. Tese de Doutorado (Doutorado em Aquicultura) - Universidade Estadual Paulista, 1997.

LOVSHIN, L.L. Modelos de desenvolvimento em piscicultura: as experiências asiática e norte-americana. In: SIMPÓSIO SOBRE MANEJO E NUTRIÇÃO DE PEIXES, 1., 1997, Piracicaba. Anais... Piracicaba: Colégio Brasileiro de Nutrição Animal, 1997. p.31-44.

LOVSHIN, L.L. Red tilapia or Nile tilapia: Wich is the best culture fish? In: SIMPÓSIO SOBRE MANEJO E NUTRIÇÃO DE PEIXES, 2., 1998, Piracicaba. Anais... Piracicaba: Colégio Brasileiro de Nutrição Animal, 1998. p.179-198. 
LOVSHIN, L.L.; CYRINO, J.E.P. Status of commercial fresh water fish culture in Brazil. In: SIMPÓSIO SOBRE MANEJO E NUTRIÇÃO DE PEIXES, 2., 1998, Piracicaba. Anais... Piracicaba: CBNA, 1998. p.1-20.

MACINTOSH, D.J.; LITTLE, D. C. Nile tilapia (Oreochromis niloticus) In: BROMAGE, N.R.; ROBERTS, R.J. (Eds.) Broodstock management and egg and larval quality. London: Blackwell Science Ltda., 1995. p.277-320.

MCANDREW, B.J. Sex control in tilapiines In: MUIR, J.F.; ROBERTS, R.J. (Eds.) Recent advances in aquaculture IV. London: Blackwell Scientific Plublications, 1993.p.87-98.

MÉLARD, C. Production of a high percentage of male offspring with 17 a ethynylestradiol sex-reversed Oreochromis aureus. I-Estrogen Sex-reversal and production of F2 pseudofemales. Aquaculture, v.130, p.25-34, 1995.

MEURER, F. Digestibilidade aparente dos nutrientes e energia de alguns alimentos protéicos para juvenis de tilápia do Nilo (Orechromis niloticus L.) e efeito do processamento da ração durante a reversão sexual. Maringá: Universidade Estadual de Maringá, 2002. 57p. Dissertação de mestrado (Mestrado em Zootecnia) - Universidade Estadual de Maringá, 2002.

MEURER, F.; HAYASHI, C.; BOSCOLO, W.R. Influência do processamento da ração no desempenho e sobrevivência da tilápia do Nilo, durante a reversão sexual. Revista Brasilera de Zootecnia, v.32, n.2, p.262-267, 2003.

MEURER, F.; HAYASHI, C.; SOARES, C.M. et al. Utilização de levedura spray dried na alimentação de tilápia do Nilo (Oreochromis niloticus L.). Acta Scientiarum, v.22, n.4, p.479-484, 2000.

PANDIAN, T.J.; SHEELA, S.G. Hormonal induction of sex reversal in fish. Aquaculture, v.138, p.1-22, 1995.

PENMAN, D.J.; MCANDREW, B.J. Genetics for the management and improvement of cultured tiliapias. In: BEVERIDGE, M.C.M.; MCANDREW, B.J. (Eds.) Tilapias: biology and explotation. London: Kluwer Academic Publishers, 2000. p.227-266.

PHELPS, R.P.; POPMA, T.J. Sex reversal of tilapia. In: COSTA-PIERCE, B.A.; RAKOCY, J.E (Eds.) Tilapia Aquaculture in the Americas. Louisiana: World Aquaculture Society, 2000. v.2, p.34-59.

PIFERRER, F. Endocrine sex control strategies for the feminization of teleost fish. Aquaculture, v.197, p.229- 281, 2001.

PIFERRER, F.; DONALDSON, E.M. Dosage-dependent differences in the effect of aromatizable and nonaromatizable androgens on the resulting phenotype of coho salmon (Oncorhynchus kisutch). Fish Physiology and Biochemistry, v.9, n.2, p.145-150, 1991.

PIFERRER, F.; DONALDSON, E.M. Gonadal differentiation in coho salmon, Oncorhynchus kisutch, after a single treatment with androgen or estrogen at different stages during ontogenesis. Aquaculture, v.77, p.251-262, 1989.
PILLAY, T.V.R. Aquaculture-priciples and practices. London: Blackwell Scientific Plublications, 1990. 575p.

POPMA, T.J.; PHELPS, R.P. Status report to commercial tilápia producers on monosex fingerling productions techniques. In: AQUICULTURA BRASIL, 10., 1998, Recife. Anais... Recife: Associação Brasileira de Aquicultura, 1998. p.127-145.

POPMA, T.J.; GREEN, B.W. Aquacultural production manual: sex reversal of tilapia in earthen ponds. Research and Development Series, v.35, p.1-15, 1990.

SANCHES, L.E.F.; HAYASHI, C. Effect of feeding frequency on Nile tilapia, Oreochromis niloticus (L.) fries performance during sex reversal in hapas. Acta Scientiarum, v.23, n.4, p.871-876, 2001.

SCOTT, A.G.; PENMAN, D.J.; BEARDMORE, J.A. et al. The 'YY'supermale in Oreochromis niloticus (L.) and its potential in aquaculture. Aquaculture, v.78, p. 237-251, 1989.

SIPAÚBA-TAVARES, L.H.S. Limnologia aplicada à aqüicultura. Jaboticabal: FINEP, 1995. 70p.

SPECKER, J.L.; CHANDLEE, M.K. Methodology for estradiol treatment in marine larval and juvenile fish: uptake and clearance in summer flounder. Aquaculture, v.217, p.663-672, 2003.

STICKNEY, R.R. Status of research on tilápia In: COSTAPIERCE, B.A.; RAKOCY, J.E. (Eds.) Tilapia aquaculture in the Americas. Louisiana: World Aquaculture Society, 2000. v.2, p.21-33.

STRUSSMANN, C.A.; TAKASHIMA, F.; TODA, K. Sex differentiation and hormonal feminization in pejerrey Odontesthes bonariensis. Aquaculture, v.139, p.31-45, 1996.

TUAN, P.A.; MAIR, G.C.; LITTLE, D.C. et al. Sex determination and the feasibility of genetically male tilapia production in the Thai - Chitralada strain of Oreochromis niloticus (L.). Aquaculture, v.173, p.257-269, 1999.

UNIVERSIDADE FEDERAL DE VIÇOSA - UFV. SAEG Sistema para Análises Estatísticas e Genéticas. Versão, 7.1. Viçosa, MG: 1997. 150p. (Manual do usuário).

YAMAMOTO, T. Sex differentiation In: HOAR, W.S.; RANDALL, D.J. (Eds.) Fish physiology. New York: Academic Press, v.3. 1969. p.117-175.

Recebido em: 05/09/03

Aceito em: 01/09/04 